\title{
Reuna
}

\section{IDENTIFICANDO A ESPIRITUALIDADE NAS ORGANIZAÇÕES}

\section{IDENTIFYING THE SPIRITUALITY IN ORGANIZATIONS}

http://dx.doi.org/10.21714/2179-8834/2019v24n1p21-40

\section{Edivandro Luiz Tecchio}

Universidade Federal da Fronteira Sul (UFFS), Brasil.

E-mail: edivandro@gmail.com

\author{
Josiane Luisa Brand \\ Instituto Federal de Santa Catarina (IFSC), Brasil. \\ E-mail: josilbrand@gmail.com \\ Lilian Rohrbeck \\ Universidade Federal da Fronteira Sul (UFFS), Brasil. \\ E-mail: lilianchapeco@hotmail.com
}

\author{
Sara Lucca \\ Universidade Federal da Fronteira Sul (UFFS), Brasil. \\ E-mail: sara.lucca@hotmail.com
}

Submissão: 19 Abr. 2018 Publicação: 05 Set. 2019. Sistema de avaliação: Double blind review. Centro Universitário UNA, Belo Horizonte - MG, Brasil. Editor geral: Prof. Dr. Gustavo Quiroga Souki

Este artigo encontra-se disponível nos seguintes endereços eletrônicos:

http://revistas.una.br/index.php/reuna/article/view/1025

http://dx.doi.org/10.21714/2179-8834/2019v24n1p21-40

\section{Resumo}

O objetivo desta pesquisa é identificar até que ponto a espiritualidade está presente em organizações de base tecnológica. Ancorado no paradigma funcionalista, conduziu-se uma survey, com corte transversal. A análise dos dados ocorreu por meio de estatística descritiva (distribuição de frequências, medidas de tendência central e de dispersão). Os resultados demonstram em que medida a espiritualidade está presente nas organizações pesquisadas. Os valores obtidos com a determinação das medidas de tendência central, em especial, a média, com valor relativamente alto, demonstra que as dimensões da espiritualidade nas organizações estão presentes nas empresas pesquisadas. Os valores das medidas de dispersão apontam que a média obtida não é fruto de fatores discrepantes. Portanto, a espiritualidade está presente no ambiente organizacional das empresas pesquisadas e seu nível pode ser considerado relativamente alto.

Palavras-chave: Espiritualidade. Espiritualidade nas Organizações. Organizações de Base Tecnológica. 


\section{ABSTRACT}

The objective this research is identify the extent to which spirituality is present in technology-based organizations. Based on the functionalist paradigm, it was conducted a survey, with cross-sectional. The data analysis occurred through descriptive statistics (distribution of frequencies, measures of central tendency and dispersion). The results demonstrate the extent to which spirituality is present in the organizations surveyed. The values obtained with the determination of the measures of central tendency, especially, the mean, with relatively high value, shows that the dimensions of spirituality in organizations are present in the companies surveyed. The values of the dispersion measurements indicate that the mean obtained is not the result of discrepant factors. Therefore, spirituality is present in the organizational environment of the companies surveyed and its level can be considered relatively high.

Keywords: Spirituality. Spirituality in Organizations. Technology-based Companies.

\section{Introduction}

As pessoas são complexas, compostas de razão, emoção e espírito. Durante muito tempo as investigações e as ações desenvolvidas nas organizações focaram sua atenção quase que exclusivamente na razão, descuidando o fato dos seres humanos serem dotados também de emoções (REGO; CUNHA; SOUTO, 2007). Os seres humanos são racionais, mas também espirituais. O corpo, a mente e o espírito integram e compõem o ser humano. Conforme apontam Ashmos e Duchon (2000, p. 136), "as pessoas possuem mente e espírito" e "o desenvolvimento do espírito é tão importante quanto o desenvolvimento da mente".

Esse entendimento a respeito do ser humano, além de outros fatores como: a) a busca de soluções espirituais para aliviar as tumultuadas mudanças sociais e de negócios (CASH; GRAY, 2000); b) as mudanças globais, que trouxeram uma crescente consciência espiritual (DUCHON; PLOWMAN, 2005); c) a necessidade de criação de um ambiente humanista; d) o entendimento de que trabalhadores felizes são mais produtivos, criativos e satisfeitos com a organização (GARCIA-ZAMOR, 2003), fazem com que o mundo coorporativo se reestruture e um "despertar espiritual" no ambiente de trabalho começou a se conformar nas últimas décadas (KHASAWNEH, 2011; GARCIA-ZAMOR, 2003).

A espiritualidade desponta como um dos aspectos principais para buscar, entre outros fatores, melhores resultados organizacionais (KHASAWNEH, 2011; FRY et al., 2011). Ela é uma abordagem relativamente nova no contexto das organizações (PARDASANI; SHARMA; BINDLISH, 2014), porém, ganhou força nos últimos anos (RAJAPPAN et. al., 2017), despertando maior interesse dos pesquisadores organizacionais sobre o tema (GARG, 2017), o que indica uma crescente importância da espiritualidade dentro do domínio organizacional (KHARI; SINHA, 2018). A espiritualidade é um fenômeno complexo, que não pode mais ser ignorado pela sociedade e pelas organizações (SANDERS; HOPKINS; GEROY, 2003). 
A estruturação de um modelo sistemático de pesquisa pode ajudar a legitimar os estudos sobre a espiritualidade nas organizações e fornecer subsídios para os gestores tomarem decisões e resolverem problemas organizacionais (HEATON; SCHMIDT-WILK; TRAVIS, 2004). Em outras palavras, é necessário estabelecer e testar formas de identificação. Conforme apontam Ashmos e Duchon (2000), desenvolver o conceito e instrumentos de mensuração da espiritualidade nas organizações é importante para que os cientistas organizacionais possam compreender e observar esse fenômeno.

Nesse sentido, o objetivo deste estudo é identificar até que ponto a espiritualidade está presente em organizações de base tecnológica. Tem-se a consciência que a pesquisa sobre a espiritualidade nas organizações está em estágio inicial, em especial, se for considerado o contexto brasileiro e, este estudo é uma forma de contribuir para a identificação da espiritualidade no ambiente laboral. É neste quadro que se insere o presente artigo. Ele está estruturado da seguinte maneira: 1) começa-se por discutir a definição de espiritualidade nas organizações; 2) elenca-se como ela é estudada; 3) após a discussão teórica apresenta-se a metodologia de pesquisa, os resultados do estudo e a análise destes; 4) por fim, elencam-se as conclusões, as limitações do estudo e sugerem-se algumas linhas para investigações futuras.

\section{Fundamentação teórica}

\subsection{Espiritualidade nas Organizações}

O termo espiritualidade, na teoria, é abstrato e subjetivo, mas na prática se apresenta como objetivo e concreto (KUMAR, 2018). Ele deriva da palavra espírito, que vem do Latim spiritu. A espiritualidade possui suas raízes fundamentais na psicologia, na religião e na filosofia (NANDRAM; BORDEN, 2010) e seu significado pode variar de acordo com cada pessoa (CAVANAGH; BANDSUCH, 2002). Nesse estudo entende-se que a espiritualidade é o desejo de encontrar um propósito final para vida e viver em consonância com ele (MITROFF; DENTON; 1999). Ela significa "as paixões nobres e reflexivas da vida e uma vida vivida em conformidade com essas paixões e reflexões nobres", ou seja, ela é "o amor reflexivo à vida". Ela abarca o amar, a confiança, a reverência e a sabedoria, bem como os aspectos mais terríveis da vida, como a tragédia e a morte (SOLOMON, 2003).

A espiritualidade não está relacionada com a crença em um Deus e não se restringe a ela (SOLOMON, 2003), ou seja, espiritualidade e religião são distintas e essa diferença está estabelecida na literatura sobre o tema (AYOUN; ROWE; YASSINE, 2015). A religião se preocupa com um sistema de crenças, orações rituais, ritos, cerimônias e práticas formais. A espiritualidade, por outro lado, preocupa-se com as qualidades do espírito humano (FRY; SLOCUM Jr., 2008). O termo espiritualidade inclui conceitos psicológicos positivos, como amor, paciência, compaixão, tolerância, perdão, contentamento, responsabilidade pessoal e uma sensação de harmonia com o meio ambiente (FRY, 2003). 
A espiritualidade apresenta os seguintes elementos-chave: não é formal, organizada ou estruturada; está acima de denominações religiosas; é amplamente inclusiva, inclui e aceita todo tipo de pessoa, não sendo proselitista; é universal, atemporal; é uma fonte essencial e provedora de significado e propósito para a vida; é o temor que se sente na presença do transcendental, o sagrado no todo, no cotidiano de cada dia; é o sentimento profundo de interconexão com o todo; é a paz interna e calma; é uma fonte inesgotável de poder e vontade; é a essência final (ultima finalidade em si) (MITROFF; DENTON; 1999).

Ao se levar a espiritualidade para o ambiente organizacional, verifica-se que ela se caracteriza por ter um aspecto universal, ou seja, por características encontradas nas mais diversas culturas e organizações (REAVE, 2005) e, apresenta uma ligação cada vez mais próxima com os conceitos de bem-estar individual e social (HARRINGTON; PREZIOSI; GOODEN, 2001; DUCHON; PLOWMAN, 2005). O conceito de espiritualidade nas organizações inclui os aspectos ou condições organizacionais, bem como mecanismos que facilitam aos trabalhadores vivenciar a espiritualidade no trabalho (PAWAR, 2008). Existem muitas formas possíveis de conceituar a espiritualidade nas organizações (KARAKAS, 2010; GARG, 2017). Até mesmo porque, ela é um fenômeno complexo, diverso, idiossincrático e multifacetado (DO, 2018), o que contribui para que não exista na literatura uma definição apropriada do que ela seja (AHMAD; OMAR, 2016). Na Tabela 1, apresenta-se uma série de conceitos encontrados na literatura.

Tabela 1: Conceitos de Espiritualidade nas Organizações

\begin{tabular}{ll}
\hline Conceito & Autor \\
\hline $\begin{array}{l}\text { O esforço de encontrar o propósito final na vida, desenvolver uma forte ligação com } \\
\text { os colegas e outras pessoas associadas com o trabalho, e ter alinhamento entre as } \\
\text { crenças individuais fundamentais e os valores de sua organização }\end{array}$ & $\begin{array}{l}\text { MITROFF; } \\
\text { DENTON, 1999 }\end{array}$ \\
\hline $\begin{array}{l}\text { O crescimento espiritual, o avanço da experiência humana, o atendimento de } \\
\text { necessidades individuais e interesses dos trabalhadores }\end{array}$ & BURACK, 1999 \\
\hline $\begin{array}{l}\text { Um quadro de valores organizacionais evidenciados na cultura que promove a } \\
\text { experiência de transcendência dos trabalhadores por meio do processo de } \\
\text { trabalho, facilitando o sentimento de conexão com os outros de uma maneira que } \\
\text { proporciona sensação de completude }\end{array}$ & $\begin{array}{l}\text { JURKIEWICZ; } \\
\text { GIACALONE, }\end{array}$ \\
\hline $\begin{array}{l}\text { Um local de trabalho que reconhece que os trabalhadores têm uma vida interior } \\
\text { que nutre e é nutrida por um trabalho significativo realizado em um contexto de } \\
\text { comunidade }\end{array}$ & $\begin{array}{l}\text { DUCHON; } \\
\text { PLOWMAN, 2005 }\end{array}$ \\
\hline $\begin{array}{l}\text { O reconhecimento de que os empregados têm uma vida interior que nutre e é } \\
\text { nutrida por um trabalho significativo que ocorre no contexto da comunidade }\end{array}$ & $\begin{array}{l}\text { ASHMOS; } \\
\text { DUCHON, 2000 }\end{array}$ \\
\hline $\begin{array}{l}\text { Uma jornada para encontrar um entendimento sustentável, autêntico, significativo, } \\
\text { holístico e profundo da auto-existência e sua relação / interligação com o sagrado e e } \\
\text { transcendente }\end{array}$ & KARAKAS, 2010 \\
\hline $\begin{array}{l}\text { Um elemento da cultura organizacional que cria um ambiente exclusivo no local de } \\
\text { trabalho e possibilita aos trabalhadores promoverem suas capacidades. }\end{array}$ & DANIEL, 2010 \\
\hline $\begin{array}{l}\text { Uma forma de expressar nossos desejos, de encontrar significado e propósito de } \\
\text { vida. É um processo de viver o conjunto de valores pessoais profundamente } \\
\text { arraigados. }\end{array}$ & $\begin{array}{l}\text { PARDASANI, } \\
\text { SHARMA. }\end{array}$ \\
\hline
\end{tabular}

Fonte: elaborado pelos autores 
Conforme relatam Rego, Souto e Cunha (2007) e também verificado por meio dos conceitos expostos (Tabela 1), arraigados nas definições de Espiritualidade nas Organizações, existem, pelo menos, três aspectos importantes: 1) os trabalhadores visam satisfazer suas necessidades espirituais, querendo ser tratados como seres únicos, estar em conexão com algo maior do que eles próprios, ser úteis, ser compreendidos e compreender como se ajustam a um contexto mais amplo, ou seja, querem um espaço para sua vida interior; 2) desejam experimentar um sentido de propósito e de significado no trabalho; e 3) desejam vivenciar um sentido de conexão com outras pessoas no ambiente laboral (senso de comunidade).

Considerando estes aspectos, o conceito adotado neste estudo é o estruturado por Ashmon e Duchon (2000), ou seja, a Espiritualidade nas Organizações é entendida como o reconhecimento de que os trabalhadores têm uma vida interior que alimenta e é alimentada por um trabalho significativo que ocorre em um contexto de comunidade. A adoção deste conceito se deve a algumas razões: 1) nesse conceito a Espiritualidade nas Organizações não está relacionada com religião; 2) Ashmon e Duchon (2000) foram pioneiros no desenvolvimento de um conceito e de um instrumento para mensuração da Espiritualidade nas Organizações; 3) seu estudo serviu de base para o desenvolvimento de diversos outros estudos; e 4) o instrumento para mensuração da Espiritualidade nas Organizações utilizado neste estudo foi estruturado por estes autores.

\subsubsection{Espiritualidade nas Organizações: como mensurar?}

Embora a espiritualidade seja um tema importante para as organizações (GIACALONE; JURKIEWICZ, 2003), seus efeitos no ambiente organizacional ainda são pouco analisados e compreendidos (MILLIMAN; CZAPLEWSKI; FERGUSON, 2003), o que demanda a execução de pesquisas, incluindo pesquisas práticas, pois "a maior parte da literatura não tem base empírica" (REGO; SOUTO; CUNHA, 2007, p. 6). Em grande medida, isso se deve ao fato de a pesquisa sobre Espiritualidade nas Organizações ter origem recente e estar em fase de formação (PAWAR, 2008). Portanto, para que seja possível compreender os efeitos da espiritualidade no ambiente de trabalho, primeiramente, é necessário realizar pesquisas empíricas de forma a identificar se a espiritualidade está presente no ambiente organizacional.

Diversos estudos foram desenvolvidos com o objetivo de identificar a presença da Espiritualidade nas Organizações (p.ex. Ashmos e Duchon (2000); Milliman, Czaplewski e Ferguson (2003); Petchsawanga e Duchon (2009); Rego e Cunha (2008); Duchon e Plowman (2005); Pawar (2009); Harrington, Preziosi e Gooden (2001); Jurkiewicz e Giacalone(2003); Kolodinsky, Giacalone e Jurkiewicz (2008); Rego, Cunha e Souto (2007); Altaf e Awan (2011);Petchsawanga e Duchon (2012); Khasawneh (2011)).

A partir da análise desses estudos, verifica-se que o método de pesquisa dominante é o quantitativo. Considerando-se este aspecto, identifica-se ainda, duas formas de mensuração: 1) por meio dos valores da Espiritualidade nas Organizações (JURKIEWICZ; GIACALONE, 2003; KOLODINSKY; GIACALONE; JURKIEWICZ, 2008); e 2) por meio das dimensões da Espiritualidade nas Organizações (ASHMOS; DUCHON, 2000; MILLIMAN; CZAPLEWSKI; FERGUSON, 2003; PETCHSAWANGA; 
DUCHON, 2009; MILLIMAN et al., 1999; REGO; CUNHA, 2008; DUCHON; PLOWMAN, 2005; PAWAR, 2009; HARRINGTON; PREZIOSI; GOODEN, 2001).

A mensuração por meio dos valores parte do pressuposto que o quadro de valores da Espiritualidade nas Organizações é definível e as organizações podem apresentá-los em diferentes níveis na sua cultura (JURKIEWICZ; GIACALONE, 2003). Para se identificar o nível em que ela está presente foi desenvolvido o Organizational Spiritual ValuesScale (OSVS). Ele é um questionário composto por diversas sentenças, as quais são avaliadas por meio de uma escala do tipo Likert, variando de 1 (completamente falsa) a 5 (totalmente verdade) (KOLODINSKY; GIACALONE; JURKIEWICZ, 2008). São poucos os estudos que utilizam este tipo de instrumento para mensurar a Espiritualidade nas Organizações.

Com mais frequência os autores utilizam as dimensões da espiritualidade para desenvolver instrumentos de mensuração (ASHMOS; DUCHON, 2000; MILLIMAN; CZAPLEWSKI; FERGUSON, 2003; PETCHSAWANGA; DUCHON, 2009; MILLIMAN et al., 1999; REGO; CUNHA, 2008; DUCHON; PLOWMAN, 2005; PAWAR, 2009; HARRINGTON; PREZIOSI; GOODEN, 2001). Esses instrumentos, basicamente, são questionários compostos pelas dimensões definidas por cada autor. Cada dimensão da espiritualidade é composta por uma série de fatores ou sentenças (ASHMOS; DUCHON, 2000; PETCHSAWANGA; DUCHON, 2009; MILLIMAN et al., 1999; REGO; CUNHA, 2008), por meio das quais os indivíduos manifestam sua opinião, em relação a cada uma delas, em uma escala numérica.

O objetivo é verificar as reações dos trabalhadores em relação a cada uma das dimensões elencadas (ASHMOS; DUCHON, 2000). Essas reações demonstram a presença de tal dimensão da espiritualidade na organização e determinam seu nível (maior ou menor intensidade). Para isso, os autores utilizam uma escala tipo Likert de 5 (cinco) (PETCHSAWANGA; DUCHON, 2009; PAWAR; 2009; KHASAWNEH, 2011) ou 7 (sete) pontos (ASHMOS; DUCHON, 2000; HARRINGTON; PREZIOSI; GOODEN, 2001; DUCHON; PLOWMAN, 2005).

A mensuração pode ser efetuada considerando três níveis: individual, unidade de trabalho (departamento, setor...) e organizacional (ASHMOS; DUCHON, 2000; HARRINGTON; PREZIOSI; GOODEN, 2001; MILLIMAN; CZAPLEWSKI; FERGUSON, 2003). Porém, os resultados em nível de unidade de trabalho e organizacional tem se revelado frustrantes como medidas da espiritualidade. Como os itens dos níveis unidade de trabalho e organizacional questionam os trabalhadores a respeito de suas relações com algo abstrato, ou seja, com a organização, a questão se torna consideravelmente mais sutil do que questioná-los acerca de si mesmos em relação a outras pessoas ou eventos em seu dia a dia no trabalho e os resultados não se mostraram satisfatórios (ASHMOS; DUCHON, 2000).

De qualquer forma, com os dados coletados, os autores utilizam de recursos estatísticos para realizar a análise destes. Entre as diversas estatísticas utilizadas encontram-se análise de correlação entre as variáveis (HARRINGTON; PREZIOSI; GOODEN, 2001; DUCHON; PLOWMAN, 2005; PAWAR, 2009; DJAFRI; NOORDIN, 2017; KHARI; SINHA, 2018), desvio padrão (KHASAWNEH, 2011; PAWAR, 2009; PETCHSAWANGA; DUCHON, 2009; DUCHON; PLOWMAN, 2005; HARRINGTON; PREZIOSI; GOODEN, 2001; ASHMOS; DUCHON, 2000), média (PAWAR, 2009; KHASAWNEH, 2011; PETCHSAWANGA; DUCHON, 2009; HARRINGTON; 
PREZIOSI; GOODEN, 2001; ASHMOS; DUCHON, 2000), análise de significância (KHASAWNEH, 2011; PETCHSAWANGA; DUCHON, 2009; ASHMOS; DUCHON, 2000; KHARI; SINHA, 2018), análise de regressão (PAWAR, 2009; ROOF 2015), regressão e correlação múltiplas (PETCHSAWANGA; DUCHON, 2009) e análise fatorial usando o método de extração de probabilidade máxima com rotação varimax (ASHMOS; DUCHON, 2000).

Como pode-se perceber, nos estudos sobre espiritualidade nas organizações utiliza-se para mensuração os valores da espiritualidade ou as dimensões desta e, após a coleta de dados são empregadas técnicas estatísticas para analisá-los. Nesse estudo, coerentemente com o exposto até aqui, para a identificação e mensuração da Espiritualidade nas Organizações serão utilizadas as chamadas dimensões da espiritualidade, as quais passam a ser descritas a seguir.

\subsubsection{Espiritualidade nas Organizações e suas dimensões}

A espiritualidade no ambiente de trabalho pode ser estudada a partir de suas dimensões (ASHMOS; DUCHON, 2000; REGO; SOUTO; CUNHA, 2007; MILLIMAN; CZAPLEWSKI; FERGUSON, 2003; PETCHSAWANGA; DUCHON, 2009). O número de dimensões varia de acordo com a abordagem de cada autor. Observa-se estudos com três (ASHMOS; DUCHON, 2000; MILLIMAN; CZAPLEWSKI; FERGUSON 2003; PAWAR, 2009; DJAFRI; NOORDIN, 2017; DEVENDHIRAN; WESLEY, 2017), quatro (PETCHSAWANGA; DUCHON, 2009), cinco (REGO; SOUTO; CUNHA, 2007; GARG, 2017) e onze (HARRINGTON; PREZIOSI; GOODEN, 2001) dimensões, que são visualizadas na Tabela 2.

Tabela 2: Dimensões da Espiritualidade nas Organizações

\begin{tabular}{ll}
\hline \multicolumn{1}{c}{ Dimensões da Espiritualidade } & \multicolumn{1}{c}{ Autores } \\
\hline Condições para comunidade & Ashmos; Duchon, 2000; Harrington; Preziosi; Gooden, \\
& 2001; Milliman; Czaplewski; Ferguson 2003; Rego; \\
& Souto; Cunha, 2007; Pawar, 2009; Faro Albuquerque \\
& et al. 2014; Afsar; Badir; Kiani,2015; Hassan; Nadeem; \\
& Akhter, 2016; Devendhiran; Wesley, 2017; Djafri; \\
& Noordin, 2017 \\
\hline Trabalho com significado & Ashmos; Duchon, 2000; Harrington; Preziosi; Gooden, \\
& 2001; Milliman; Czaplewski; Ferguson 2003; \\
& Petchsawanga; Duchon, 2009; Pawar, 2009; Faro \\
& Albuquerque et al. 2014; Afsar; Badir; Kiani,2015; \\
& Hassan; Nadeem; Akhter, 2016; Devendhiran; Wesley, \\
& 2017; Djafri; Noordin, 2017 \\
\hline Vida interior & Ashmos; Duchon, 2000; Harrington; Preziosi; Gooden, \\
& 2001; Rego; Souto; Cunha, 2007; Faro Albuquerque et \\
& al. 2014 \\
\hline Alegria no trabalho & Rego; Souto; Cunha, 2007 \\
\hline Alinhamento do indivíduo com os valores da & Milliman; Czaplewski; Ferguson 2003; Rego; Souto; \\
organização & Cunha, 2007 \\
\hline Sentido de préstimo à comunidade & Rego; Souto; Cunha, 2007 \\
\hline Compaixão & Petchsawanga; Duchon, 2009 \\
\hline Atenção plena (consciência interior) & Petchsawanga; Duchon, 2009 \\
\hline
\end{tabular}


Tabela 2: Dimensões da Espiritualidade nas Organizações (continuação)

\begin{tabular}{|c|c|}
\hline Dimensões da Espiritualidade & Autores \\
\hline Transcendência & Petchsawanga; Duchon, 2009 \\
\hline Proposito organizacional positivo & Pawar, 2009 \\
\hline Comunidade na unidade de trabalho & Harrington; Preziosi; Gooden, 2001 \\
\hline Alinhamento de valores organizacionais & $\begin{array}{l}\text { Harrington; Preziosi; Gooden, 2001; Afsar; Badir; } \\
\text { Kiani,2015; Hassan; Nadeem; Akhter, 2016; } \\
\text { Devendhiran; Wesley, 2017; Djafri; Noordin, } 2017\end{array}$ \\
\hline Valores positivos na unidade de trabalho & Harrington; Preziosi; Gooden, 2001 \\
\hline Contemplação & Harrington; Preziosi; Gooden, 2001 \\
\hline Conexões positivas com outros indivíduos & Harrington; Preziosi; Gooden, 2001 \\
\hline Responsabilidade pessoal & Harrington; Preziosi; Gooden, 2001 \\
\hline Bloqueios para a espiritualidade & Harrington; Preziosi; Gooden, 2001 \\
\hline Indivíduos e organização & Harrington; Preziosi; Gooden, 2001 \\
\hline
\end{tabular}

Fonte: elaborado pelos autores

Ao analisar a Tabela 2, observa-se que as dimensões da espiritualidade mais utilizadas na realização de estudos são: 1) senso de comunidade; 2) trabalho com significado; e 3) vida interior. Essas três dimensões são as definidas e utilizadas por Ashmos e Duchon (2000) e serviram de base para outros estudos realizados por diversos autores, inclusive as demais dimensões elencadas no referido quadro estão intimamente ligadas a essas três dimensões, como pode ser verificado a seguir.

1) Senso de comunidade: as dimensões responsabilidade pessoal, conexões positivas, comunidade no trabalho, valores organizacionais (HARRINGTON; PREZIOSI; GOODEN, 2001) já haviam sido identificadas por Ashmos e Duchon (2000) como fatores que complementam a dimensão senso de comunidade. A dimensão compaixão (PETCHSAWANGA; DUCHON, 2009) também está relacionada em grande medida a esta dimensão, já que envolve um profundo senso de conexão entre as pessoas, o que inclui suporte, liberdade de expressão, cuidado genuíno (MILLIMAN; CZAPLEWSKI; FERGUSON, 2003), responsabilidade e desejo de aliviar o sofrimento do outro, e carinho mútuo (PETCHSAWANGA; DUCHON, 2009);

2) Trabalho com significado: as dimensões valores positivos e indivíduos e organização (HARRINGTON, PREZIOSI, GOODEN, 2001) são fatores apontados por Ashmos e Duchon (2000) como sendo relacionados ao trabalho com significado. O mesmo ocorre com a dimensão préstimo à comunidade (REGO; SOUTO; CUNHA, 2007) e propósito organizacional (PAWA, 2009). Além disso, a dimensão alegria no trabalho pode ser considerada uma subdimensão do trabalho com significado (REGO; SOUTO; CUNHA, 2007);

3) Vida interior: a dimensão contemplação (HARRINGTON, PREZIOSI, GOODEN (2001), relaciona-se com comportamentos associados à expressão da vida interior (ASHMOS; DUCHON, 2000), o que também ocorre com as dimensões transcendência e atenção plena. A transcendência envolve um estado positivo de energia ou vitalidade, um sentimento de perfeição, experiências de alegria e felicidade. Já a atenção plena refere-se a mente da pessoa estar presente, não vagando com pensamentos passados, futuros ou outras distrações (PETCHSAWANGA; DUCHON, 2009). 
Portanto, como pode-se observar pela análise aqui efetuada, as dimensões 1) senso de comunidade, 2) trabalho com significado e 3) vida interior (ASHMOS; DUCHON, 2000), englobam, de alguma maneira, todas as demais dimensões. Assim, coerentemente com o conceito de espiritualidade nas organizações adotado e, considerando que essas três dimensões abarcam as demais e são as que compõem o questionário a ser utilizado para verificação da Espiritualidade nas Organizações, passa-se a discorrer sobre elas.

O senso de comunidade significa uma ligação profunda com os outros (ASHMOS; DUCHON, 2000). Esta dimensão ocorre no nível de grupo, envolvendo o comportamento, as interações e as preocupações do indivíduo para com seus colegas de trabalho. A comunidade no trabalho está amparada na crença que as pessoas veem a si mesmas como conectadas umas às outras e que há algum tipo de ligação com o "eu interior" de um indivíduo com o "eu interior" dos outros. Essencialmente, o sentido de comunidade envolve um profundo senso de conexão entre as pessoas, o que inclui suporte, liberdade de expressão e cuidado genuíno (MILLIMAN; CZAPLEWSKI; FERGUSON, 2003).

O trabalho com significado envolve um sentido profundo de significado e propósito no trabalho desenvolvido. Esta dimensão representa como os trabalhadores interagem com seu trabalho no dia a dia, em nível individual. A expressão da espiritualidade no trabalho pressupõe que cada pessoa tem suas próprias motivações, verdades e desejos de estar envolvida em atividades que forneçam maior significado, tanto para sua vida quanto para a dos outros. A busca de propósito para a vida, considerando o ambiente de trabalho, não é algo novo, porém, no contexto da espiritualidade, não basta um trabalho interessante ou desafiador, busca-se um significado mais profundo e objetivo, a vivência de um sonho, a expressão das necessidades da vida interior por meio de um trabalho com significado (ASHMOS; DUCHON, 2000).

A vida interior é um aspecto fundamental para a compreensão da espiritualidade no ambiente de trabalho e seu alimento pode levar à significativa e produtiva vida exterior (ASHMOS; DUCHON, 2000). As pessoas têm necessidades espirituais assim como possuem necessidades físicas, emocionais e cognitivas, e essas necessidades não ficam em casa quando as pessoas vão trabalhar (DUCHON; PLOWMAN, 2005). Além disso, o desenvolvimento e a expressão do espírito humano no trabalho podem levar a consequências benéficas para a organização (ASHMOS; DUCHON, 2000).

\section{Metodologia de pesquisa}

Tendo como base o paradigma funcionalista (MORGAN, 2005), este estudo segue os pressupostos da pesquisa quantitativa (SAMPIERI; COLLADO; LUCIO, 2006). Além de quantitativo, este estudo se caracteriza por ser exploratório. A pesquisa exploratória é utilizada quando o objetivo é investigar um tema que é pouco pesquisado (SAMPIERI; COLLADO; LUCIO, 2006). Em relação à estratégia de pesquisa, o estudo se caracteriza como survey (PINSONNEAULT; KRAEMER, 1993). O questionário utilizado para a coleta de dados e consequente mensuração da Espiritualidade nas Organizações foi desenvolvido por Ashmos e Duchon (2000). Este questionário é composto pelas três dimensões da Espiritualidade nas 
Organizações imbuídas no conceito definido por eles e também utilizado nesse estudo, ou seja, senso de comunidade, trabalho com significado e vida interior. Cada uma dessas dimensões é composta por uma série de sentenças culminando em 28 fatores. Para a mensuração dos fatores o questionário utiliza-se de uma escala tipo Likert de 7 (sete) pontos, onde 1 (um) representa total discordância com o fator avaliado e 7 (sete) total concordância com o fator avaliado, ou seja, $1=$ discorda totalmente e 7 = concorda totalmente. $O$ questionário estruturado encontra-se no Anexo I.

Como o questionário foi traduzido do inglês para o português efetuou-se testes de validação do questionário. A validação ocorreu em duas etapas: 1) préteste; e 2) validação da confiabilidade interna do questionário por meio da determinação do coeficiente alfa de Cronbach. Inicialmente o questionário foi apresentado a professores da área de gestão (dois professores) e linguística (dois professores). O objetivo foi verificar se os termos do questionário estavam adequados, tanto em termos da ciência da administração quanto à linguística. Após aplicou-se o questionário a trabalhadores de uma empresa que compunha a amostra. Nesse momento buscou-se identificar dificuldades em relação ao entendimento das sentenças por parte desses respondentes. Como não houve problemas, o questionário foi considerado adequado para a realização da pesquisa.

A verificação da confiabilidade interna foi efetuada por meio do cálculo do coeficiente alfa de Cronbach, desenvolvido por J. L. Cronbach (SAMPIERI; COLLADO; LUCIO, 2006). O alfa de Cronbach é um índice utilizado para avaliar a magnitude em que os itens de um instrumento estão correlacionados $(\mathrm{CRONBACH}$, 1951). Foi identificado o alfa de Cronbach para cada uma das variáveis, buscando assim identificar a consistência de seus itens. O cálculo foi efetuado considerando a fórmula definida por Cronbach (1951).

Em relação à interpretação da confiabilidade de um questionário obtida por meio do alfa de Cronbach, em geral, considera-se boa a consistência interna de um instrumento de pesquisa quando é obtido $\alpha \geq 0,70$. Conforme apontam Gliem e Gliem (2003) e Streiner (2003), o valor mínimo aceitável para o Alfa de Cronbach é 0,7 . Seguindo os pressupostos presentes na literatura, verifica-se que os coeficientes calculados apresentam-se elevados, o que é esperado e comprova a consistência interna dos fatores relacionados a cada variável de estudo, conforme pode ser verificado na Tabela 3.

Tabela 3: Coeficiente alfa de Cronbach

\begin{tabular}{lccc}
\hline Variável & Senso de Comunidade & Trabalho com Significado & Vida Interior \\
\hline Cronbach & 0,79 & 0,86 & 0,83 \\
\hline
\end{tabular}

Fonte: elaborado pelos autores

Os coeficientes obtidos são significativos. Por mais que se afirme que eles podem variar de acordo com o padrão de resposta da população estudada, estudos desenvolvidos sobre o tema (p.ex.: Rego; Cunha e Souto (2007), Petchsawanga e Duchon (2009), Altaf e Awan (2011), Harrington, Preziosi e Gooden (2001), Tombaugh, Mayfield e Durand (2011), Liu e Robertson (2010), Milliman, Czaplewski e Ferguson (2003), Rego e Cunha (2008), Petchsawanga e Duchon (2012)) REINA, Belo Horizonte - MG, Brasil, v.24, n.I, p.2I-4D, Jan. - Мar. 2019 - ISSN 2179-8834 
apresentam valores semelhantes desse coeficiente para os questionários desenvolvidos para mensurar a Espiritualidade nas Organizações o que, em grande medida, fornece segurança na utilização e aplicação desse questionário. Portanto, considera-se o questionário validado.

A aplicação do questionário se deu de forma presencial, durante o horário de expediente, considerando os pressupostos do corte transversal (SAMPIERE; COLLADO; LUCIO, 2006). Ela ocorreu junto a trabalhadores de Empresas de Base Tecnológica, com sede no município de Chapecó-SC, associadas ao Polo Tecnológico do Oeste Catarinense, núcleo vinculado a Associação Catarinense de Empresas de Tecnologia. Ao todo, 20 empresas participaram da pesquisa, totalizando 133 (cento e trinta e três) respondentes.

A análise dos dados se deu por meio da estatística descritiva, onde foram determinadas estatísticas como média, mediana, moda, desvio padrão, variância e coeficiente de variação. Essas medidas foram obtidas por meio do software SPSS StatisticalPackage for the Social Sciences. O SPSS é um software estatístico desenvolvido pela IBM voltado, principalmente, para pesquisadores da área de ciências sociais. Ele possui uma infinidade de funções e possibilita as mais diversas análises estatísticas, as quais atendem as necessidades do estudo.

\section{Discussão dos resultados}

A pesquisa foi realizada com 133 trabalhadores de 20 (vinte) empresas de base tecnológica situadas no município de Chapecó, Santa Catarina, associadas ao Polo Tecnológico do Oeste Catarinense - DEATC, núcleo vinculado a ACATE Associação Catarinense de Empresas de Tecnologia. Estas empresas são consideradas Organizações Intensivas em Conhecimento. Elas atuam no ramo de desenvolvimento de software, portanto, classificam-se por atuar no setor de comércio e prestação de serviços e são caracterizadas como micro e pequenas empresas.

Os trabalhadores dessas empresas em sua maioria são do sexo masculino (75\%) e apresentam uma faixa etária baixa. $74 \%$ possuem menos de 30 anos, sendo que a maioria, $62 \%$, possui entre 21 e 30 anos. Em sua maioria são solteiros, com um total de $49 \%$. $21 \%$ são casados e $30 \%$ vivem em união estável com seus/suas parceiros/parceiras. Em relação a escolaridade, em sua maioria os respondentes possuem ensino superior completo, sendo que, $28 \%$ possui graduação, $24 \%$ especialização e $1 \%$ mestrado. O total de respondentes que possui nível superior é de $53 \%$, ou em números absolutos 70 respondentes.

\subsection{Nível de Espiritualidade nas Organizações pesquisadas}

Após a coleta dos dados, as respostas obtidas foram tabuladas e organizadas na forma de distribuição de frequências para a obtenção das frequências relativas. Para cada respondente foi estabelecido um fator único relativo para cada variável, representado o nível daquela variável. Assim, cada variável tem seu respectivo valor, que varia de um (mínimo) a sete (máximo). Para se chegar a esse valor, foram somados os valores atribuídos pelos respondentes aos indicadores desta e dividido 
pelo total de indicadores. Esses valores representam, o nível de cada dimensão da espiritualidade nas organizações. No Apêndice $B$, apresenta-se a distribuição de frequência com os valores relativos a cada variável, de acordo com a percepção de cada respondente.

Com base nesses valores estabeleceu-se as medidas de tendência central, uma vez que elas representam os fenômenos pelos valores médios, em torno dos quais tendem a se concentrar os dados. Na Tabela 5 encontram-se os resultados relativos a média, mediana e moda de cada variável da Espiritualidade nas Organizações.

Tabela 5: Medidas de tendência central das variáveis da espiritualidade nas organizações.

\begin{tabular}{lccc}
\hline & Senso de Comunidade & Trabalho com Significado & Vida Interior \\
\hline Média & 5,62 & 5,24 & 5,46 \\
\hline Mediana & 5,71 & 5,29 & 5,57 \\
\hline Moda & 5,36 & 4,86 & 5,57 \\
\hline
\end{tabular}

Fonte: Dados primários

A análise dos valores médios, presentes na Tabela 5, possibilita inferir que os respondentes consideram que, nas organizações onde trabalham, estão presentes fatores relacionados ao senso de comunidade, a realização de um trabalho com significado e condições que possibilitam o desenvolvimento da vida interior. Considerando a escala de medição, pode-se afirmar que o nível em que cada variável da espiritualidade nas organizações está presente no ambiente de trabalho é elevado.

No entanto, essas medidas de tendência central, em especial a média, podem ser "viciadas" por dados discrepantes presentes em qualquer questionário. Nesse sentido, é importante calcular as medidas de dispersão. As medidas de dispersão demonstram como os dados tendem a se dispersar em torno de um valor médio e a heterogeneidade dos valores (BARBETTA, 2007). Nesse sentido, verifica-se, pelas medidas de dispersão, apresentadas na Tabela 6 , que os valores da variância e do desvio padrão são relativamente baixos, o que demonstra pouca heterogeneidade dos dados em termos absolutos, ou seja, eles tendem à homogeneidade.

Tabela 6: Desvio padrão e variância das variáveis relacionadas a espiritualidade nas organizações

\begin{tabular}{lccc}
\hline & Senso de Comunidade & Trabalho com Significado & Vida Interior \\
\hline Desvio padrão & 0,68 & 0,87 & 1,04 \\
\hline Variância & 0,46 & 0,76 & 1,07 \\
\hline
\end{tabular}

Fonte: dados primários

Analisando o desvio padrão das variáveis, observa-se que dentre todas as variáveis a que apresenta a menor dispersão absoluta é senso de comunidade, com 0,68 pontos e a que apresenta a maior é vida interior, com 1,04 pontos. No entanto, 
a variância e o desvio padrão são medidas de dispersão absolutas. Para entender melhor o significado da dispersão dos dados é importante identificar a dispersão relativa. Essa medida relativa é obtida por meio do cálculo do coeficiente de variação - CV (FONSECA; MARTINS, 2009).

O coeficiente de variação é uma medida de dispersão relativa, apresentado em percentuais (FONSECA; MARTINS, 2009). Para determinar se a dispersão é baixa, média ou alta, os autores determinam faixas, onde enquadram os valores do coeficiente de variação calculados. Segundo Fonseca e Martins (2009), alguns analistas, e esse é o entendimento adotado neste estudo, consideram baixa dispersão: $C V \leq 15 \%$, média dispersão: $15 \%<C V<30 \%$ e alta dispersão: $C V \geq 30 \%$. Com base nos valores da Tabela 7 observa-se que a dispersão relativa dos valores das variáveis é baixa ou média. O que demonstra que eles giram em torno da média e tendem a homogeneidade.

Tabela 7: Coeficiente de variação

\begin{tabular}{cccc}
\hline & Senso de Comunidade & Trabalho com Significado & Vida Interior \\
\hline Coeficiente de variação & $12 \%$ & $16 \%$ & $19 \%$ \\
\hline
\end{tabular}

Fonte: dados primários

Portanto, analisando-se as medidas descritivas aqui apresentadas, verifica-se que os valores obtidos com a determinação das medidas de tendência central, em especial, a média, com valor relativamente alto, não é fruto de fatores discrepantes. Ela demonstra que os valores obtidos para cada dimensão da espiritualidade nas organizações estão dispersas homogeneamente nas organizações estudadas, reforçando sua importância, sugerindo ainda, como já exposto, que o nível de espiritualidade pode ser considerado elevado, o que remete a uma série de implicações, conforme discutido a seguir.

\subsection{Discussão}

Conceituar a Espiritualidade nas Organizações com a maior clareza possível e desenvolver formas para identificá-la/mensurá-la é importante para que os pesquisadores organizacionais possam compreender e observar esse fenômeno (ASHON; DUCHON, 2000). Os resultados da validação do questionário por meio do pré-teste e da verificação da confiabilidade interna, por meio da determinação do coeficiente alfa de Cronbach, atestam a validade do instrumento e fornecem segurança à pesquisa desenvolvida, impactando, inclusive, na confiabilidade dos resultados encontrados, os quais sugerem que a espiritualidade está presente nas organizações pesquisadas.

A presença da espiritualidade nessas organizações traz uma série de implicações, pois reconhecer a espiritualidade no ambiente de trabalho é admitir que as pessoas levam consigo mais do que seus corpos e mentes. As organizações que ao longo do tempo ignoraram esse fato básico, inerente à natureza humana, agora exploram conceitos espirituais como confiança, harmonia, valores e honestidade com o intuito de alcançar seus objetivos ( $\mathrm{LEIGH}, 1997)$. A espiritualidade recebe 
importante atenção, a ponto de ser colocada como fundamental para enfrentar os desafios do ambiente de trabalho (MITROFF; DENTON, 1999).

Estando a espiritualidade presente nas organizações pesquisadas, uma série de benefícios podem ser desencadeados, os quais impactam no desempenho organizacional (DUSCHON; PLOWMAN, 2005; GARCIA-ZAMOR, 2003; REGO; SOUTO; CUNHA, 2007; KHASAWNEH 2011; PAWAR, 2009; ROBLES et al., 2011; KARAKAS, 2010; MILLIMAN; CZAPLEWSKI; FERGUSON, 2003; ALTAF; AWAN, 2011; JURKIEWICZ; GIACALONE, 2004). A maioria das pesquisas considera que a espiritualidade no ambiente de trabalho proporciona tanto benefícios para os trabalhadores quanto para a organização (SANTIAGO, 2007). Na Tabela 8 elenca-se alguns dos benefícios apontados pela literatura como sendo decorrentes de um ambiente organizacional espiritualizado.

Tabela 8: Benefícios da Espiritualidade nas Organizações

\section{BENEFÍCIOS}

\section{AUTOR}

\begin{tabular}{|c|c|}
\hline $\begin{array}{l}\text { Diminuição dos vícios no local de trabalho, } \\
\text { sentido de significado e propósito, senso } \\
\text { de comunidade, aumento do bem-estar e } \\
\text { lealdade. }\end{array}$ & Karakas, 2010 \\
\hline $\begin{array}{l}\text { Aumento da moral e senso de } \\
\text { pertencimento a organização. }\end{array}$ & Garcia-Zamor, 2003; Karakas, 2010 \\
\hline Aumento da produtividade e da criatividade & $\begin{array}{l}\text { Garcia-Zamor, } 2003 \text { e 2004; Karakas, 2010; Altaf; } \\
\text { Awan, 2011; Duschon; Plowman, 2005; Rego; Souto; } \\
\text { Cunha, 2007 }\end{array}$ \\
\hline Diminuição do stress & Karakas, 2010; Altaf; Awan, 2011 \\
\hline $\begin{array}{l}\text { Maior comprometimento com a } \\
\text { organização }\end{array}$ & $\begin{array}{l}\text { Milliman; Czaplewski; Ferguson, 2003; Karakas, 2010; } \\
\text { Altaf; Awan, 2011; Jurkiewicz; Giacalone, 2004; } \\
\text { Duschon; Plowman, 2005; Garcia-Zamor, 2003; Rego; } \\
\text { Souto; Cunha, 2007; Djafri; Noordin, 2017; Milliman; } \\
\text { Gatling; Kim, } 2018\end{array}$ \\
\hline Aumento da felicidade & Garcia-Zamor, 2003; Duschon; Plowman, 2005 \\
\hline Maior satisfação com o trabalho & $\begin{array}{l}\text { Milliman; Czaplewski; Ferguson, 2003; Altaf; Awan, } \\
\text { 2011; Duschon; Plowman, 2005; Garcia-Zamor, 2003; } \\
\text { Rego; Souto; Cunha, } 2007\end{array}$ \\
\hline $\begin{array}{l}\text { Maior envolvimento com o trabalho e } \\
\text { aumento da autoestima }\end{array}$ & Milliman; Czaplewski; Ferguson, 2003 \\
\hline Menor índice de rotatividade & $\begin{array}{l}\text { Milliman; Czaplewski; Ferguson, 2003; Altaf; Awan, } \\
\text { 2011; Duschon; Plowman, 2005; Rego; Souto; Cunha, } \\
2007\end{array}$ \\
\hline $\begin{array}{l}\text { Melhora nas relações pessoais, na } \\
\text { comunicação, na tomada de decisão e no } \\
\text { desempenho, aumento da confiança, do } \\
\text { comportamento ético e da realização } \\
\text { pessoal e mais inovação. }\end{array}$ & Altaf; Awan, 2011 \\
\hline Maior motivação e adaptabilidade & Jurkiewicz; Giacalone, 2004 \\
\hline Redução do absenteísmo & Duschon; Plowman, 2005 \\
\hline Compartilhamento de conhecimento & Rahman, 2015; Khari; Sinha, 2018 \\
\hline $\begin{array}{l}\text { Bem-estar emocional, psicológico, social e } \\
\text { espiritual. }\end{array}$ & Pawar, 2016 \\
\hline
\end{tabular}

Fonte: elaborado pelos autores

RELUNA, Belo Horizonte - MG, Brasil, v.24, n.I, p.2I-4D, Jan. - Мar. 2019 - ISSN 2179-8834 
Em que pese os possíveis benefícios proporcionados pela Espiritualidade nas Organizações favorecerem tanto as organizações quanto as pessoas que nela trabalham é necessário analisar de forma crítica para que a espiritualidade não seja utilizada de forma manipulada. Enquanto existem ganhos potenciais significativos que levam ao fomento da espiritualidade na organização, existe também uma preocupação em relação a sua utilização de forma manipulada, visando somente ganhos organizacionais, em detrimento aos possíveis benefícios que ela pode trazer aos trabalhadores (CANAVAGH; BANDSUCH, 2002; MILLIMAN; CZAPLEWSKI; FERGUSON, 2003). Quando a organização adota práticas que promovem a espiritualidade, estes aspectos devem ser considerados. A espiritualidade deve prover benefícios tanto para a organização quanto para os indivíduos que nela trabalham.

\section{Considerações finais}

Este estudo procurou contribuir com uma área que tem emergido com força, principalmente, fora do Brasil, mas que ainda é alvo de pouca pesquisa empírica: a Espiritualidade nas Organizações. O tema é controverso (KARAKAS, 2010; GARCIA-ZAMOR, 2003). Durante muito tempo as investigações e as ações desenvolvidas nas organizações focaram sua atenção quase que exclusivamente na razão, descuidando do fato de os seres humanos serem dotados também de emoções (REGO; CUNHA; SOUTO, 2007). A integração da variável "espiritualidade" nos estudos organizacionais, indica uma evolução do paradigma adotado pela ciência administrativa de um extremo ao outro (KHARI; SINHA, 2018). Com essa evolução é possível afirmar que as organizações entendem que, além de racionais os seres humanos são também espirituais (ASHMOS; DUCHON, 2000). Sendo os seres humanos, também, espirituais, que procuram significado para suas vidas por meio do trabalho realizado em um contexto de comunidade, as organizações que não promovem as condições para que isso ocorra poderão ser penalizadas, inclusive, com desempenho individual e, consequentemente, organizacional, menor do que esperado (REGO; CUNHA; SOUTO, 2007). Portanto, para as organizações é importante saber se a espiritualidade está presente ou não em seu ambiente e compreender quais as implicações que isso apresenta para a sua gestão.

Nesse sentido, esse estudo procurou identificar se a espiritualidade estava presente nas organizações de base tecnológica pesquisadas. Os resultados apontam para a presença da espiritualidade no ambiente organizacional das empresas pesquisadas e seu nível pode ser considerado relativamente alto. Um alto índice de Espiritualidade nas Organizações pode proporcionar uma série de benefícios como já elencado anteriormente, mas também uma grande responsabilidade para com os trabalhadores. Existe uma preocupação ética sobre a incorporação da espiritualidade no ambiente de trabalho, no sentido de que ela é usada como uma ferramenta administrativa para manipular os trabalhadores (DENT; HIGGINS; WHARFF, 2005). Quando se incorpora a espiritualidade no local de trabalho, deve-se ter em mente uma mudança de cultura organizacional, para que os trabalhadores, nos diversos níveis hierárquicos e, a organização como um todo, passem a adotar, no seu dia a dia, práticas e políticas humanistas (GARCIAZAMOR, 2003). 
É importante destacar que, sendo a espiritualidade o meio pelo qual os trabalhadores procuram encontrar um significado mais profundo para a vida. As organizações devem reconhecer que, juntamente com o estresse e os problemas no local de trabalho, outros aspectos da vida social afetam os trabalhadores e estes procuram caminhos para enfrentá-los (HARRINGTON; PREZIOSI; GOODEN, 2001). A Espiritualidade nas Organizações é colocada como um dos caminhos para enfrentar tais situações e, além disso, aumentar o desempenho organizacional (ASHMOS; DUCHON, 2000; KARAKAS, 2010; ALTAF; AWAN, 2011; KHASAWNEH 2011; FRY et al.; 2011). Portanto, a Espiritualidade nas Organizações é uma via de mão dupla e, ao incentivá-la no ambiente organizacional os benefícios que ela proporciona precisam contemplar a organização como um todo, em seus diversos aspectos.

Por fim, ressalta-se que novos estudos devem ser desenvolvidos. A espiritualidade deve ser relacionada com os mais diversos aspectos da vida organizacional, a fim de identificar seu real impacto para a organização e a vida dos trabalhadores, bem como confirmar os benefícios que ela pode proporcionar. Apesar de sua identificação no ambiente laboral ser fundamental para os estudos que envolvem o tema, estudar seus impactos e como ela se relaciona com os mais diversos aspectos da vida organizacional é essencial para que ela proporcione benefícios a todos os integrantes da organização.

\section{Referências}

AFSAR, Bilal; BADIR, Yuosre; KIANI, Umar Safdar. Linking spiritual leadership and employee pro-environmental behavior: The influence of workplace spirituality, intrinsic motivation, and environmental passion. Journal of Environmental Psychology, v. 45, p. 79-88, 2015.

AHMAD, Aminah; OMAR, Zoharah. Workplace spirituality among Malaysian community service employees in the public sector. Asian Social Science, v. 12, n. 9 , p. 193-201, 2016.

ALTAF, Amal; AWAN, Mohammad Atif. Moderating Affect of Workplace Spirituality on the Relationship of Job Overload and Job Satisfaction. Journal Business Ethics. 104. 2011.

ASHMOS, Donde P.; DUCHON, Dennis. Spiritualityatwork: conceptualizationandmeasure. Journalof Management Inquiry. v. 9. nํㅡ. 2. 2000.

AYOUN, Baker; ROWE, Louis; YASSINE, Fatima. Is workplace spirituality associated with business ethics?. International journal of Contemporary hospitality management, v. 27, n. 5, p. 938-957, 2015.

BURACK, Elmer H. Spirituality in the workplace. Journal of Organizational Change Management. vol. 12. n4, 1999. 
CASH, Karen C.; GRAY, George R. A framework for accommodating religion and spirituality in the workplace. The Academy of Management Executive, v. 14, n. 3, p. $124-133,2000$.

CAVANAGH, Gerald F.; BANDSUCH, Mark R. Virtue as a benchmark for spirituality in business. Journal of Business Ethics, v. 38, n. 1-2, p. 109-117, 2002.

CRONBACH, Lee J. Coefficient alpha and the internal structure of tests. Psychometrika, v. 16, n. 3, p. 297-334, 1951.

DANIEL, Jose Luis. The effect of workplace spirituality on team effectiveness. Journal of Management Development. v. 29. nำ 5. 2010.

DENT, Eric B.; HIGGINS, T, M. Eileen; WHARFF, Deborah M. Spirituality and leadership: an empirical review of definitions, distinctions, and embedded assumptions. The Leadership Quarterly. vol. 16, 2005.

DEVENDHIRAN, Sangeetha; WESLEY, J. Reeves. Spirituality at work: enhancing levels of employee engagement. Development and Learning in Organizations: An International Journal, v. 31, n. 5, p. 9-13, 2017.

DJAFRI, Fares; NOORDIN, Kamaruzaman. The impact of workplace spirituality on organizational commitment: A case study of Takaful agents in Malaysia. Humanomics, v. 33, n. 3, p. 384-396, 2017.

DO, Tung Thanh. How spirituality, climate and compensation affect job performance. Social Responsibility Journal, v. 14, n. 2, p. 396-409, 2018.

DUCHON, Dennis; PLOWMAN, DondeAshmos. Nurturing the spirit at work: Impact on work unit performance. TheLeadershipQuarterly. v. 16. 2005.

FARO ALBUQUERQUE, Isabel et al. Primary health care services: workplace spirituality and organizational performance. Journal of Organizational Change Management, v. 27, n. 1, p. 59-82, 2014.

FONSECA, Jairo Simon da; MARTINS, Gilberto de Andrade. Curso de estatística. 6 ed. Atlas: São Paulo, 2009.

FRY, Louis W. Toward a theory of spiritual leadership. The leadership quarterly, v. 14, n. 6, p. 693-727, 2003.

FRY, Louis W. et al. Impact of spiritual leadership on unit performance. The Leadership Quarterly. vol. 22, 2011.

FRY, Louis W. SLOCUM, John W. Jr. Maximizing the Triple Bottom Line through Spiritual Leadership. Organizational Dynamics. v. 37, no 1. Elsevier, 2008.

GARCIA-ZAMOR, Jean-Claude. Workplace Spirituality and Organizational Performance. Public Administration Review. vol. 63, n 3. 2003. 
GARG, Naval. Workplace spirituality and employee well-being: An empirical exploration. Journal of Human Values, v. 23, n. 2, p. 129-147, 2017.

GLIEM, Joseph A.; GLIEM, Rosemary R. Calculating, interpreting, and reporting Cronbach's alpha reliability coefficient for Likert-type scales. Midwest Research-toPractice Conference in Adult, Continuing, and Community Education, 2003.

HARRINGTON, William J.; PREZIOSI, Robert C.; GOODEN, Doreen J. Perceptions of workplace spirituality among professionals and executives, Employee Responsibilities \& Rights Journal. v. 13 nํ 3. 2001.

HASSAN, Misbah; BIN NADEEM, Ali; AKHTER, Asma. Impact of workplace spirituality on job satisfaction: Mediating effect of trust. Cogent Business \& Management, v. 3, n. 1, p. 1189808, 2016.

HEATON, Dennis P.; SCHMIDT-WILK, Jane; TRAVIS, Frederick. Constructs, methods, and measures for researching spirituality in organizations. Journal of organizational change management, v. 17, n. 1, p. 62-82, 2004.

JURKIEWICZ, Carole L.; GIACALONE, Robert A. A Values Framework for Measuring the Impact of Workplace Spirituality on Organizational Performance. Journal of Business Ethics. vol. 49, 2004.

KARAKAS, Fahri. Spirituality and Performance in Organizations: a Literature Review. Journal of Business Ethics. v. 94. 2010.

KHARI, Chitra; SINHA, Shuchi. Organizational Spirituality and Knowledge Sharing: A Model of Multiple Mediation. Global Journal of Flexible Systems Management, v. 19, n. 4, p. 337-348, 2018.

KHASAWNEH, Samer. Cutting-edge panacea of the twenty-first century: Workplace spirituality for higher education human resources. International Journal of Educational Management. vol. 25 nํ 7, 2011.

KOLODINSKY, Robert W.; GIACALONE, Robert A.; JURKIEWICZ, Carole L. Workplace Values and Outcomes: Exploring Personal, Organizational, and Interactive Workplace Spirituality. Journal of Business Ethics. v. 81. 2008.

KUMAR, Sunil. A study of perceived workplace spirituality of school teachers. Psychological Thought, v. 11, n. 2, p. 212-223, 2018.

MILLIMAN, John; CZAPLEWSKI, Andrew J.; FERGUSON, Jeffery. Workplace spirituality and employee work attitudes: an exploratory empirical assessment. Journal of Organizational Change Management. vol. 16 ํㅜ 4, 2003.

MILLIMAN, John; GATLING, Anthony; KIM, Jungsun Sunny. The effect of workplace spirituality on hospitality employee engagement, intention to stay, and service delivery. Journal of Hospitality and Tourism Management, v. 35, p. 56-65, 2018. 
MITROFF, Ian I.; DENTON, Elizabeth A. A spiritual audit of corporate America: A hard look at spirituality, religion, and values in the workplace. Jossey-Bass, 1999.

MORGAN, Gareth. Paradigmas, metáforas e resolução de quebra-cabeças na teoria das organizações. RAE. v. 45, nº 1. 2005.

NANDRAM, Sharda S.; BORDEN, Margot Esther. Introduction: Exploring possibilities for a new paradigm. In: Spirituality and business. Springer Berlin Heidelberg, 2010. p. 1-13.

PARDASANI, Rupali; R. SHARMA, Radha; BINDLISH, Puneet. Facilitating workplace spirituality: Lessons from Indian spiritual traditions. Journal of Management Development, v. 33, n. 8/9, p. 847-859, 2014.

PAWAR, Badrinarayan Shankar. Individual spirituality, workplace spirituality and work attitudes: an empirical test of direct and interaction effects. Leadership \& Organization Development Journal. vol. 30. №. 8, 2009.

Workplace spirituality and employee well-being: An empirical examination. Employee Relations, v. 38, n. 6, p. 975-994, 2016.

PETCHSAWANGA, Pawinee; DUCHON, Dennis. Measuring workplace spiritualit y in an Asian context. Human Resource Development International. v. 12. nำ 4. 2009.

. Workplace spirituality, meditation, and work performance. Journal of Management, Spirituality \& Religion, v. 9, n. 2, p. 189-208, 2012.

PINSONNEAULT, Alain; KRAEMER, Kenneth L. Survey Research Methodology in Management Information Systems: An Assessment. Journal of Management Infromation System, 1993.

RAHMAN, Muhammad Sabbir et al. Trust and work place spirituality on knowledge sharing behaviour: Perspective from non-academic staff of higher learning institutions. The Learning Organization, v. 22, n. 6, p. 317-332, 2015.

RAJAPPAN, Smitha et al. Exploring the effect of workplace spirituality on job embeddedness among higher secondary school teachers in Ernakulam district. Cogent Business \& Management, v. 4, n. 1, p. 1334419, 2017.

REGO, Arménio; CUNHA, Miguel Pinha; SOUTO, Solange. Espiritualidade nas Organizações e Comprometimento Organizacional. RAE eletrônica, v. 6, №. 2, 2007.

REGO, Arménio; CUNHA, Miguel Pinha. Workplace spirituality and organizational commitment: an empirical study. Journal of Organizational Change Management. v. 21 no. $1,2008$. 
ROOF, Richard A. The association of individual spirituality on employee engagement: The spirit at work. Journal of Business Ethics, v. 130, n. 3, p. 585-599, 2015.

SANDERS, Joseph E.; HOPKINS, Willie E.; GEROY, Gary D. From transactional to transcendental: Toward an integrated theory of leadership. Journal of Leadership \& Organizational Studies, v. 9, n. 4, p. 21-31, 2003.

SAMPIERI, Roberto Hernández; COLLADO, Carlos Fernández; LUCIO, Pilar Baptista. Metodología de la Investigación. 4aㅡ ed. Ciudad de México. México: McGraw-Hill, 2006.

STREINER, David L. Being inconsistent about consistency: When coefficient alpha does and doesn't matter. Journal of personality assessment, v. 80 , n. 3, p. 217222, 2003.

SOLOMON, Robert C. Espiritualidade para céticos: paixão, verdade cósmica e racionalidade no século XXI. Civilização Brasileira. Rio de Janeiro, 2003. 